\title{
Proceso de atención de enfermería a un paciente pediátrico en el transcurso de una biopsia renal
}

\author{
José Arcioles-Fernández ${ }^{1}$, Isabel María Oliver-Jiménez ${ }^{2}$, M $^{a}$ Luisa Sánchez-Martínez ${ }^{3}$, Concepción Amat-Medina ${ }^{4}$ \\ ${ }^{1}$ Hospital Comarcal Minas de Rio Tinto SAS. Huelva. España \\ ${ }^{2}$ Instituto Social de la Marina.Huelva. España \\ ${ }^{3}$ Servicio de Prevención Riesgos laborales MPE. Huelva. España \\ ${ }^{4}$ Unidad Materno Infantil Hospital de Inca Mallorca. España
}

Como citar este artículo:

Arcioles Fernández J, Oliver Jiménez IM, Sánchez Martínez ML, Amat Medina C. Proceso de atención de enfermería a un paciente pediátrico en el transcurso de una biopsia renal.

Enferm Nefrol. 2020 Jul-Sep;23(3):303-310

\section{Resumen}

Descripción del caso: Paciente de 13 años que ingresa en hospital de tercer nivel, procedente de consultas externas de Nefrología pediátrica del mismo hospital para biopsia renal, al sufrir, en valoraciones anteriores, episodios de hematurias microscópicas persistentes, macroscópicas intermitentes y proteinuria en rango nefrótico.

Descripción del plan de cuidados: La unidad de enfermería pediátrica del hospital estableció un plan de cuidados inicial al ingreso y postquirúrgico ajustados a la patrones funcionales de Marjory Gordon, considerando los patrones relacionados con temor en el niño, ansiedad y cavilación de la familia, resultando de estos hechos la activación del protocolo de acogida, aumento del nivel de estrés debido a modificación ingesta alimentos, aumentar el afrontamiento en la fase postquirúrgica así como dolor, riesgo de infección, disconfort, sobrepeso por alimentación insana y hematurias postbiopsia.

Evaluación del plan: Durante el ingreso hospitalario de 4 días previo a la biopsia se le realizaron pruebas bioquímicas de sangre y orina de 24 horas y estudio de anemia. Los progenitores se mostraban muy interesados por la evolución de las pruebas y como contener la ansiedad y tener calmado al hijo debido al conocimiento de que le harían una punción. Mientras se encontraba hospitalizado no cumplía los requisitos nutricionales. Al

\section{Correspondencia:}

José Arcioles Fernández

Email: jorioles@correo.ugr.es día siguiente de la biopsia fue dado de alta con unas pautas para comenzar con una alimentación más sana.

Conclusiones: La taxonomía NANDA/NOC/NIC permite trabajar en la enfermería basada en los hallazgos, dando respuesta a las necesidades reales de los pacientes para contribuir a mejorar la práctica clínica.

PALABRAS CLAVE: síndrome nefrótico; hematuria; NANDA/NOC/NIC; biopsia renal; patrones funcionales.

\section{Nursing care process to a paediatric patient during a renal biopsy}

\section{Abstract}

Clinical case: A 13-year-old patient admitted to a thirdlevel hospital from paediatric nephrology outpatient clinics for a renal biopsy after suffering, in previous evaluations, episodes of intermittent macroscopic and persistent microscopic haematuria and nephrotic-range proteinuria.

Description of the care plan: The hospital paediatric nursing unit established an initial care plan on admission and after surgery adjusted to Gordon's functional patterns, considering the patterns related to fear in the child, anxiety and family melancholy, resulting in the activation of the reception protocol, increased stress level due to food intake modification, increased coping in the post-surgical phase as well as pain, risk 
of infection, discomfort, overweight due to unhealthy eating and post-biopsy haematuria.

Evaluation of the care plan: During the 4-day hospital stay prior to the biopsy, 24-hour blood and urine biochemical tests and anemia study were performed. The parents were very interested in the evolution of the tests and how to contain anxiety and keep the child calm due to the knowledge that they would do a blood puncture. While he was hospitalized, he did not meet the nutritional requirements. The day after the biopsy, he was discharged with guidelines to start a healthier diet.

Conclusions: The NANDA/NOC/NIC taxonomy allows working in nursing based on findings, responding to the real needs of patients to help improve clinical practice.

KEYWORDS: Nephrotic syndrome; haematuria; NANDA/NOC/NIC; kidney biopsy; functional patterns.

\section{Introducción}

Una de las técnicas que más han contribuido al conocimiento de las enfermedades renales ha sido la biopsia renal y aunque es una técnica invasiva, no suele haber complicaciones, aunque no está exenta de ciertos riesgos ${ }^{1}$.

La biopsia renal consiste en la extracción mediante una aguja especial de una muestra milimétrica de tejido renal. La punción se realiza bajo control ecográfico con anestesia local con o sin sedación, según las características del paciente, posteriormente. La muestra obtenida es procesada por el Servicio de Anatomía Patológica con el uso de diferentes técnicas para la observación bajo microscopia óptica, microscopia de fluorescencia y microscopia electrónica. Así se pueden observar los diferentes compartimentos del riñón: los glomérulos, los túbulos y los vasos sanguíneos².

El objetivo de la misma es evaluar el sangrado persistente en orina o la proteinuria y el diagnóstico puede dar lugar a una neuropatía ${ }^{3}$ confirmado con un estudio anatomopatológico a través del análisis de una muestra de tejido renal por microscopía óptica e inmunofluorescencia. Las indicaciones de biopsia renal (BR) se basan, entre otras, en la edad, manifestaciones clínicas, evolución, respuesta al tratamiento, y dentro de esta última, la corticorresistencia en el síndrome nefrótico ${ }^{4}$.

El paciente objeto de la biopsia dispone de un historial sobre su situación clínica y el objeto de su ingreso unos días antes de la biopsia no es más que para confirmar los datos históricos que se han venido recopilando unos meses antes, con pruebas específicas a efectuar en el momento del ingreso tales como un estudio de anemia, anticuerpos antinucleares (ANAS), anti DNA, anticitoplasma de neutrófilos (ANCAS) y una ecografía renal que permita conocer situación y tamaño, diferenciación corticomedular y posibilidad de dilatación de vías excretoras y existencias de quistes corticales.

Las anomalías urinarias mínimas (enfermedad de cambio mínimos) se presentan en pacientes que manifiestan enfermedad renal solamente por análisis de orina patológico y como manifestaciones patológicas se pueden observar hematuria, proteinuria, cilindruria, glucosuria, cristaluria, piuria ${ }^{1}$.

\section{Presentación del caso}

Paciente de 13 años de edad, que presenta desde hace 6 meses episodios de hematurias macroscópicas de aproximadamente 12 horas de evolución, a veces más intensas tras ejercicio, proteinuria en rango nefrótico, sin sintomatología, astenia importante, (absentismo escolar intermitente) pérdida de peso no cuantificable y tensión arterial normal. Ingresa en un hospital de tercer nivel, área de influencia de Andalucía occidental, procedente de consultas externas de nefrología pediátrica del mismo hospital, para biopsia renal. Sin antecedentes familiares de enfermedad renal.

A su ingreso se le han realizado numerosas pruebas complementarias destacando, proteinuria en rango nefrótico persistente con discreta hipoproteinemia, hematuria microscópica persistente y macroscópica intermitente, orina de 24 horas, valores de proteinuria de $71 \mathrm{mg} / \mathrm{m}^{2} / \mathrm{h}$ y 5,4 g/L proteínas totales. Estudio de coagulación normal. Ecografía renal, riñones situados en su posición habitual y de tamaño normal. Buena diferenciación corticomedular y sin dilatación de vías excretoras. Riñón derecho presenta un quiste cortical a nivel del polo superior de $12,9 \mathrm{~mm}$. Todos estos valores no hacen más que confirmar los resultados obtenidos en los últimos meses programándose la biopsia renal debido a lo inadmisible de la perdida de proteínas y según resultados establecer un tratamiento de choque que frene dicha perdida.

\section{Valoración inicial enfermera al ingreso}

La enfermera de la Unidad de nefrología pediátrica del hospital realiza una valoración según los patrones funcio- 
nales de Marjory Gordon ${ }^{5}$, para el desarrollo del proceso enfermero se sigue la taxonomía diagnóstica de NANDA/NOC/NIC.

La encuesta de valoración inicial enfermera (Tablal) detectó una actitud de temor y estrés relacionada con el nuevo entorno, la biopsia y ansiedad ante la incertidumbre de los resultados. En cuanto a la nutrición mostró rechazo a la comida del hospital y poca ingesta de líqui-

Tabla 1. Diagnósticos (NANDA), resultados (NOC) e intervenciones (NIC).

\begin{tabular}{|c|c|}
\hline $\begin{array}{l}\text { Percepción/ } \\
\text { Manejo salud. }\end{array}$ & $\begin{array}{l}\text { Vacunaciones completes, no hay patologías } \\
\text { crónicas, sin dificultades familia/paciente } \\
\text { para seguir con el tratamiento. }\end{array}$ \\
\hline $\begin{array}{l}\text { Nutrición/ } \\
\text { metabolismo. }\end{array}$ & $\begin{array}{l}\text { Sin dificultad para la alimentación, autó- } \\
\text { nomo, sin dificultad para tragar, beber y } \\
\text { masticar, mucosa oral sin alteración, peso } \\
51,5 \mathrm{Kg} \text {, talla } 1,60 \text { e IMC } 20,1 \text {. } \\
\text { Durante el ingreso se niega a comer la co- } \\
\text { mida del hospital y exige a los progenitores } \\
\text { comida del exterior. Bebe poco liquido } \\
\text { Piel hidratada, palidez y sin edemas. }\end{array}$ \\
\hline Eliminación. & $\begin{array}{l}\text { Patrón habitual normal sin ayuda farmaco- } \\
\text { lógica, autónomo, aunque no elimina desde } \\
\text { hace } 24 \text { horas. Eliminación urinaria sin } \\
\text { alteración. }\end{array}$ \\
\hline $\begin{array}{l}\text { Actividad/ } \\
\text { Ejercicio. }\end{array}$ & $\begin{array}{l}\text { Respiración normal, ausencia de tos y } \\
\text { ruidos respiratorios, autónomo en la } \\
\text { deambulación. }\end{array}$ \\
\hline Reposo/Sueño. & $\begin{array}{l}\text { Su patrón habitual son } 8 / 9 \text { horas de sueño } \\
\text { sin ayuda farmacológica. }\end{array}$ \\
\hline $\begin{array}{l}\text { Cognitivo/ } \\
\text { Perceptivo. }\end{array}$ & $\begin{array}{l}\text { Comunicación verbal completa, consciente, } \\
\text { comprensión de los datos sensoriales que } \\
\text { conduce a la identificación, asociación y/o } \\
\text { el reconocimiento de patrones. }\end{array}$ \\
\hline $\begin{array}{l}\text { Autopercep- } \\
\text { ción/autocon- } \\
\text { cepto. }\end{array}$ & Su carácter habitual es tranquilo. \\
\hline Rol/ relaciones. & Su cuidador principal son sus progenitores. \\
\hline $\begin{array}{l}\text { Sexualidad/ } \\
\text { reproducción. }\end{array}$ & Desarrollo normal órganos sexuales. \\
\hline $\begin{array}{l}\text { Afrontamiento } \\
\text { tolerancia al } \\
\text { estrés. }\end{array}$ & $\begin{array}{l}\text { La actitud del niño ante la enfermedad y el } \\
\text { ingreso hospitalario es temerosa y ansie- } \\
\text { dad y los padres manifiestan un cuadro de } \\
\text { ansiedad. }\end{array}$ \\
\hline
\end{tabular}

dos, inadecuada en la escala de medición, exigiendo a sus padres comida procedente del exterior.

\section{Plan de cuidados}

Durante el período de ingreso se han valorado como diagnósticos enfermeros (DE), Temor, Ansiedad y Riesgo de síndrome de estrés del traslado.

La Tabla 2 muestra los diagnósticos NANDA, los resultados NOC así como las intervenciones NIC que han tenido lugar durante el ingreso hospitalario, tanto en el periodo previo como posterior a la biopsia renal.

Con relación a la ansiedad al ingreso, se aplica un protocolo de acogida tanto para el niño como para padres, se explican las dudas sobre hospitalización, permitir que desahoguen sus preocupaciones, enfatizar sobre los beneficios de un buen diagnóstico para un correcto tratamiento y se justo con el niño y explicarle las incomodidades que va sentir.

La Tabla 3 muestra la valoración de la hoja de enfermería hospitalaria desde el ingreso hasta el alta.

\section{Evaluación del Plan}

Aunque el progreso tras la biopsia fue bien, por el color de las orinas no se observaron hematurias macroscópicas en la primera y siguientes orinas postbiopsia, las tiras reactivas en orina $\left(\right.$ Labsti $\left.^{\circledR}\right)$ confirmaban la presencia de microhematuria, situación habitual en las horas posteriores a la biopsia renal. Durante la tarde y noche presentó un cuadro de intranquilidad sintiéndose incómodo. Se le administró $5 \mathrm{mg}$ de tranxilium que le permitió relajarse hasta el día siguiente, procurando la familia crear un ambiente confortable. El apósito que cubría la incisión presentó un aspecto limpio sin manchas de sangre. Estuvo afebril, sin calor ni dolor en la zona de punción.

El temor y la ansiedad fueron disminuyendo paulatinamente a través de la escucha activa, reconociendo y ayudando al joven a reconocer las situaciones que aceleran la angustia y el miedo. Con relación al estrés debido al cambio de entorno e ingreso hospitalario, el paciente no fue preparado previamente lo que provocó una necesidad de preguntar continuamente si sus hermanos habían venido a verlo e indicaciones exactas de la dirección donde se encontraba hospitalizado, dismi- 
Tabla 2. Diagnósticos NANDA, resultados NOC e intervenciones NIC previos y tras la biopsia renal.

\begin{tabular}{|c|c|c|}
\hline NANDA $^{6}$ & $N_{O C}{ }^{7}$ & NIC $^{8}$ \\
\hline $\begin{array}{l}\text { (00148) Temor relacionado con }(\mathrm{r} / \mathrm{c}) \\
\text { con entorno desconocido, susceptibili- } \\
\text { dad percibida elevada y manifestada } \\
\text { por }(\mathrm{m} / \mathrm{p}) \text { nerviosismo, angustia, voz } \\
\text { temblorosa, disminución en la seguridad } \\
\text { de sí mismo }\end{array}$ & $\begin{array}{l}\text { (1404). Autocontrol del miedo } \\
\text { Indicadores: } \\
\text { (140403).Control del miedo, el niño } \\
\text { pide información para reducir el miedo. } \\
\text { Nivel de miedo infantil }\end{array}$ & $\begin{array}{l}\text { (5340) Presencia } \\
\text { Actividades: } \\
\text { Escuchar las preocupaciones del hijo } \\
\text { Establecer una consideración de con- } \\
\text { fianza positiva entre padres e hijo. } \\
\text { ( } 4920 \text { ) Escucha activa } \\
\text { Actividades: } \\
\text { Mostrar interés por lo que expresa el } \\
\text { niño } \\
\text { Utilizar el silencio/escucha para animar } \\
\text { a expresar sus sentimientos, pensamien- } \\
\text { tos y preocupaciones. }\end{array}$ \\
\hline $\begin{array}{l}\text { (00146) Ansiedad, r/c situación de } \\
\text { salud y entorno y } \mathrm{m} / \mathrm{p} \text { crisis de angustia, } \\
\text { cavilación y preocupación a causa de } \\
\text { cambios vitales. }\end{array}$ & $\begin{array}{l}\text { (1300) Aceptación: estado de salud. } \\
\text { Acciones personales para reconciliar los } \\
\text { cambios significativos en las circunstan- } \\
\text { cias de salud. (130017) Se adapta al } \\
\text { cambio en el estado de salud. } \\
\text { (1402) Autocontrol de la ansiedad. } \\
\text { Acciones personales para eliminar o re- } \\
\text { ducir sentimientos de aprensión, tensión } \\
\text { o inquietud de una fuente no identifica- } \\
\text { da. (140204) El niño pide información } \\
\text { para reducir la ansiedad. } \\
\text { (1402) Autocontrol de la ansiedad. } \\
\text { Acciones personales para eliminar o re- } \\
\text { ducir sentimientos de aprensión, tensión } \\
\text { o inquietud de una fuente no identifica- } \\
\text { da. (140204) Busca información para } \\
\text { reducir la ansiedad. }\end{array}$ & $\begin{array}{l}\text { (4920) Escucha activa: Prestar gran } \\
\text { atención y otorgar importancia a los } \\
\text { mensajes verbales y no verbales del } \\
\text { niño. } \\
\text { (5820) Minimizar la aprensión, temor, } \\
\text { presagios o inquietud relacionados con } \\
\text { una fuente no identificada de peligro } \\
\text { previsto, aún no sabe la realización } \\
\text { de la biopsia renal. Ayudar al niño a } \\
\text { identificar las situaciones que precipitan } \\
\text { la ansiedad. }\end{array}$ \\
\hline $\begin{array}{l}\text { (00149) Riesgo de síndrome de estrés } \\
\text { del traslado. } \\
\text { Vulnerable a sufrir un trastorno } \\
\text { psicológico tras un cambio de entorno } \\
\text { r/c aislamiento de su entorno y amigos } \\
\text { habituales } \mathrm{m} / \mathrm{p} \text { inseguridad y desconoci- } \\
\text { miento del medio }\end{array}$ & $\begin{array}{l}\text { (1301) Adaptación del niño a la hospi- } \\
\text { talización. (130112). Buena relación } \\
\text { padres-hijo excepto en lo concerniente } \\
\text { a la alimentación. (130123). Pregunta } \\
\text { por su enfermedad. }\end{array}$ & $\begin{array}{l}\text { (5440) Se aumentó los sistemas de } \\
\text { apoyo, fundamentalmente (5270) el } \\
\text { emocional. } \\
\text { Proporcionar actividades recreativas. }\end{array}$ \\
\hline $\begin{array}{l}\text { (00047) Riego de deterioro de la } \\
\text { integridad cutánea definido por riesgos } \\
\text { de que la piel se vea negativamente } \\
\text { afectada r/c procedimiento quirúrgico } \\
\text { invasivo. }\end{array}$ & $\begin{array}{l}\text { (01101). Mantener la integridad tisular. } \\
\text { (11010) Ausencia de lesión tisular. } \\
\text { (110113) La piel se encuentra intacta. }\end{array}$ & $\begin{array}{l}\text { (3590) Vigilancia de la piel, comprobar } \\
\text { apósito limpio, que no haya fuentes de } \\
\text { presión y fricción, mantener, reposo en } \\
\text { decúbito supino, se vigila color, calor in- } \\
\text { flamación y edema en la zona punzada. }\end{array}$ \\
\hline $\begin{array}{l}\text { (0005) Riesgo de desequilibrio de la } \\
\text { temperatura corporal r/c intervención } \\
\text { quirúrgica y } \mathrm{m} / \mathrm{p} \text { subida temperatura } \\
\text { corporal. }\end{array}$ & $\begin{array}{l}\text { (0800) Termorregulación resolver } \\
\text { durante la estancia. (080001) Tempe- } \\
\text { ratura cutánea aumentada, nivel leve. } \\
(080019) \text {. Hipertermia leve. }\end{array}$ & $\begin{array}{l}\text { Actividades: (390006) comprobar la } \\
\text { temperatura corporal cada } 15 \text { minutos } \\
\text { tras biopsia e ir espaciando, ( } 390007) \text {. } \\
\text { Controlar tensión arterial. ( } 390018) \\
\text { registrar temperaturas. }\end{array}$ \\
\hline $\begin{array}{l}\text { (D.E):(00234) Riesgo de sobrepeso, } \\
\text { vulnerable a la acumulación de grasa } \\
\text { excesiva para la edad y sexo que puede } \\
\text { comprometer su salud r/c comer de } \\
\text { restaurantes o fritos habitualmente y } \\
\mathrm{m} / \mathrm{p} \text { patrones de comidas disfunciona- } \\
\text { les, que no cumplen con sus requisitos } \\
\text { nutricionales. }\end{array}$ & $\begin{array}{l}\text { (1855) Estilo de vida saludable. } \\
\text { (184109) Practicas nutricionales sa- } \\
\text { ludable. (184112) Antojo de alimentos } \\
\text { que desencadenan una alimentación no } \\
\text { saludable. }\end{array}$ & $\begin{array}{l}\text { (1100) Manejo de la nutrición, propor- } \\
\text { cionar y fomentar una ingesta equilibra- } \\
\text { da de nutrientes. } \\
\text { (1160) Monitorización nutricional, de- } \\
\text { terminar los patrones de comidas (p.ej., } \\
\text { gustos y aversiones alimentarias, consu- } \\
\text { me exceso de comida rápida, comidas no } \\
\text { realizadas. interacción entre progenitores } \\
\text { y el niño durante la alimentación. }\end{array}$ \\
\hline
\end{tabular}




\begin{tabular}{|c|c|c|}
\hline NANDA $^{6}$ & NOC $^{7}$ & NIC $^{8}$ \\
\hline $\begin{array}{l}\text { Problema interdependiente (P.I.): } \\
\text { P.I.: Riesgo de hematurias macroscópica } \\
\text { postbiopsia. }\end{array}$ & $\begin{array}{l}\text { Observar color de la orina durante } 24 \\
\text { horas siguientes a la punción. }\end{array}$ & $\begin{array}{l}\text { Control constantes vitales como la ten- } \\
\text { sión arterial (TA), frecuencia cardiaca } \\
\text { (FC) y temperatura cada } 4 / 8 \text { horas } \\
\text { Control hematocrito según prescripción } \\
\text { médica. } \\
\text { Control del color de la orina hasta } 24 \\
\text { horas después y avisar al facultativo en } \\
\text { caso de seguir sangrando. } \\
\text { Vigilar síntomas de hipovolemia } \\
\text { Registrar y evaluar la presencia de } \\
\text { sangre en cada micción a lo largo de las } \\
24 \text { horas siguientes. }\end{array}$ \\
\hline $\begin{array}{l}\text { P.I.: Riesgo de infección por microor- } \\
\text { ganismos patógenos r/c procedimiento } \\
\text { invasivo punción percutánea y acceso } \\
\text { venoso periférico. }\end{array}$ & Vigilar integridad tisular, piel intacta. & $\begin{array}{l}\text { Cuidados de las heridas. Drenaje cerra- } \\
\text { do, desinfectar con yodopovidona. } \\
\text { Cuidados del lugar de incisión. (apósito } \\
\text { limpio). } \\
\text { Cuidados del catéter, Controlar el punto } \\
\text { de fijación del catéter. Buena higiene de } \\
\text { manos para su manipulación. }\end{array}$ \\
\hline $\begin{array}{l}\text { (DE) } 00214 \text {. Disconfort, falta de } \\
\text { tranquilidad, alivio y transcendencia } \\
\text { desde el punto de vista físico y psíquico, } \\
\text { relacionado con sentirse incómodo. }\end{array}$ & $\begin{array}{l}\text { 2002) Bienestar personal. } \\
\text { (2008) Estado de comodidad. }\end{array}$ & $\begin{array}{l}\text { Administrar medicación. } \\
\text { (6482) Manejo ambiental, creando un } \\
\text { ambiente tranquilo y de apoyo, mejorar } \\
\text { la posición del catéter, la ropa de cama } \\
\text { arrugada y apoyos con almohadas. }\end{array}$ \\
\hline
\end{tabular}

Tabla 3. Valoración en la hoja de enfermería.

\begin{tabular}{|c|c|c|c|}
\hline \multicolumn{4}{|l|}{$\underline{1}^{\circ}$ Día de ingreso: } \\
\hline & Mañana & Tarde & Noche \\
\hline Alimentación: & -/Comida de la calle & - & $-/-$ \\
\hline Labstix: & - & - & - \\
\hline Deposiciones: & - & - & - \\
\hline Diuresis: & $250 c c$ & $600 c c$ & 100 \\
\hline Área orgánica: & asintomatico/normotenso & asint./normot. & asint./normot \\
\hline Área psicoafectiva: & tranquilo & tranquilo & descansa \\
\hline Temperatura/Pulso/TA: & $35,5^{\circ} \mathrm{C} / 73 \mathrm{ppm} / 131-81 \mathrm{mmHg}$ & $36^{\circ} \mathrm{C} / 70 \mathrm{ppm} / 109-66 \mathrm{mmHg}$ & $36,1^{\circ} \mathrm{C} / 86 \mathrm{ppm} / 111-76 \mathrm{mmHg}$ \\
\hline \multicolumn{4}{|l|}{$\underline{2}^{\circ}$ Día de ingreso: } \\
\hline & Mañana & Tarde & Noche \\
\hline Alimentación: & Bien/Comida calle & -/Comida de la calle & $-1-$ \\
\hline Labstix: & Glucosa -/Cet- & - & - \\
\hline Deposiciones: & - & - & - \\
\hline Diuresis: & $200 c c$ & $250 c c$ & $200 c c$ \\
\hline Área orgánica: & asint./normot. & asint./normot. & asint./normot. \\
\hline Área psicoafectiva: & alegre & tranquilo & descansa \\
\hline Temperatura/Pulso/TA: & $36,5^{\circ} \mathrm{C} / 79 \mathrm{ppm} / 114-75 \mathrm{mmHg}$ & $36,5^{\circ} \mathrm{C} / 85 \mathrm{ppm} / 115-65 \mathrm{mmHg}$ & $36^{\circ} \mathrm{C} / 88 \mathrm{ppm} / 129-75 \mathrm{mmHg}$ \\
\hline
\end{tabular}




\begin{tabular}{|c|c|c|c|}
\hline \multicolumn{4}{|l|}{$3^{\circ}$ Día de ingreso: } \\
\hline & Mañana & Tarde & Noche \\
\hline Alimentación: & Bien/Comida calle & $-1-$ & - \\
\hline Labstix: & proteinuria: +++/hematuria++++pH5 & - & - \\
\hline Deposiciones: & - & - & - \\
\hline Diuresis: & 100cc (hematuria) & $200 c c$ & $750 \mathrm{cc}$ \\
\hline Área orgánica: & asint./normot. & asint./normot. & asint./normot. \\
\hline Área psicoafectiva: & tranquilo & tranquilo & nervioso \\
\hline Temperatura/Pulso/TA: & $36,6^{\circ} \mathrm{C} / 73 \mathrm{ppm} / 125-75 \mathrm{mmHg}$ & $36,5^{\circ} \mathrm{C} / 77 \mathrm{ppm} / 100-60 \mathrm{mmHg}$ & $36,6^{\circ} \mathrm{C} / 74 \mathrm{ppm} / 118-70 \mathrm{mmHg}$ \\
\hline
\end{tabular}

\section{$4^{\circ}$ Día de ingreso: Biopsia renal}

9:30. Medicación matinal: Suero glucosalino 2000cc/24h; desmopresina ( 3 ampollas de 4 microgramos en 200 cc Suero glucosalino en 3 horas); Midazolan 0,5 mg.

Valores previos: TA122/65 mm Hg, FC 101ppm. Valores postbiopsia;inmediatamente: TA 131/74 mmHg, FC 104 ppm, a los 30 minutos: TA 113/70 mmHg, FC 100 ppm, a los 60 minutos: TA 115/70 mmHg, FC: 87ppm.

Mañana

Alimentación:

Labstix:

Deposiciones:

Diuresis:

Área orgánica:

\section{Mañana:}

Valorar cada micción macroscopicamente (hematuria). En caso de 2 orinas hematuricas seguidas se informa, hacer hemograma urgente y avisar al nefrólogo de guardia.

Observaciones: Regresa de la biopsia a las 14:00 h, mantiene reposo absoluto en decubito supino hasta las 18:00 horas, posteriormente se podrá mover ligeramente en la cama hasta hacer las 24 horas transcurridas desde a punción. Apósito limpio. No levantarse.

\section{Tarde:}

Afebril, reposo relativo en cama a partir de las 18:00 $\mathrm{h}$

$1^{a}$ orina no se observa hematuria macroscópica.

No vuelve a orinar.

Área psicoafectiva: Muy nervioso e inquieto, faltando el respeto a sus progenitores. Se le administra 17:00 h tranxilium (5mgr) vía oral, ya que por se niega a que se lo inyecten. Dieta blanda por la tarde y para cenar dieta normal. Resto de la tarde tranquilo.

Temperatura/Pulso/TA: $35,5^{\circ} \mathrm{C} / 91 \mathrm{ppm} / 120-80 \mathrm{mmHg}$.

\section{Noche:}

Área orgánica:

Normotenso. No precisa tranxilium. Orina no hematúrica (macroscópica)

Área psicoafectiva: muy tranquilo, semidormido, colaborador

Temperatura/Pulso/TA: $36,5^{\circ} \mathrm{C} / 83 \mathrm{ppm} / 100-69 \mathrm{mmHg}$.

\section{$\underline{5^{\circ} \text { Día de ingreso: Alta }}$}

$\begin{array}{lc} & \text { Mañana } \\ \text { Alimentación: } & \text { Bien / - } \\ \text { Labstix: } & \text { hematuria }++++ \\ \text { Deposiciones: } & \text { no } \\ \text { Diuresis: } & 500 \mathrm{cc} \\ \text { Temperatura/Pulso/TA: } & 36,5^{\circ} \mathrm{C}, /-/ 124 / 67 \mathrm{mmHg}\end{array}$

cc: centímetros cúbicos; ${ }^{\circ} \mathbf{C}$ : grados centígrados; ppm: pulsaciones por minuto; $\mathbf{m m H g}$ : milímetros de mercurio, TA: tensión arterial. 
nuyendo el riesgo hasta desaparecer mediante el apoyo emocional de sus padres, proporcionando algunas actividades recreativas.

Se produjo una buena recuperación y a las 24 horas fue dado de alta con objeto de que una vez se confirmado el diagnóstico se pudiera iniciar el tratamiento más adecuado.

Otro aspecto a destacar fue el riesgo de sobrepeso por malos hábitos alimentarios que derivaron en ansiedad y estrés. Se le prescribió con el alta un plan de alimentación por escrito, que se explicó tanto a progenitores como al niño, basado fundamentalmente en una dieta baja en sal, limitando la cantidad de proteínas de origen animal carne/pescado, fomentando el consumo proteínas de bajo valor biológico, restringiendo el consumo de lácteos y productos derivados de huevo.

\section{Discusión}

La biopsia renal percutánea es un procedimiento con bajo riesgo, en el que la mayoría de las complicaciones son menores y no tienen repercusiones clínicas, pero para ello es necesario llevar una disciplina en el procedimiento, evitando factores de riesgo y vigilando la aparición de posible complicacciones?. Una de las complicaciones más serias que se puede presentar es la hemorragia, que en algunos casos va a precisar transfusión, y por ello en las hojas de valoración de enfermería (Tabla 3) se insiste en la valoración de macrohematuria en micción postbiopsia, que si se presenta en dos micciones consecutivas debe informarse al nefrólogo y realizar un hemograma urgente. En el caso de pacientes con microhematurias, como en el caso que se describe, hay que esperar un diagnóstico de biopsia renal, para proceder a un tratamiento médico. La importancia de monitorizar la tensión arterial durante el ingreso y postbiopsia junto con el estudio de coagulación, ecografía renal y urocultivo estéril y consentimiento informado son requisitos mínimos para realizar la biopsia renal ${ }^{10}$.

Durante la valoración inicial en el momento del ingreso, la enfermera evaluó la necesidad de una correcta alimentación, observando desde un primer momento malos hábitos alimentarios a pesar de tener talla, peso e IMC correcto y aquí la labor enfermera debe valorar las características definitorias, y no solo por lo que se puede apreciar mediante observación directa y medir, sino por lo que el paciente/familia cuenta junto con los trastornos psicológicos de temor y estrés ambiental que pueden mermar el apetito. Las actividades de la intervención pasaron por determinar las preferencias de comidas del niño, pero buscando un equilibrio nutricional, interaccionando para ello padres e hijo, recompensando objetivos cumplidos.

La falta de satisfacción con la situación de ingreso pudo deteriorar la salud física y mental del niño, desequilibrando el estado de ánimo y su capacidad para el afrontamiento. Por tanto, es importante detectar falta de fuerza de voluntad en el mismo, por lo que es prioritario para enfermería fortalecer este concepto creando un buen ambiente, estableciendo prioridades, y a partir de lo que está pasando, ayudar a el paciente desarrolle buenos conocimientos sobre la situación, que contribuya a fortalecerse y para ello necesita apoyo e información de su familia y del conjunto de la comunidad médico-sanitaria.

A partir de los resultados encontrados podemos concluir que los diagnósticos enfermeros basados en la taxonomía NANDA permiten trabajar en la enfermería basada en los hallazgos, que a su vez proporcionan un lenguaje universal a los profesionales para diagnosticar y obtener resultados a través de la planificación del plan de cuidados, permite comunicar con otros profesionales de la salud, e interactuar con pacientes y familias. La efectividad de los cuidados aplicados al niño en general han sido satisfactorios y en lo que se refiere a los diagnósticos que requieren datos subjetivos del paciente como ansiedad/temor o disconfort podrían tratarse de temor/ansiedad o afrontamiento ineficaz difícil de dilucidar con exactitud, aunque la valoración minuciosa en profundidad en estos casos es fundamental.

Recibido: 19-11-19

Revisado: 14-01-20

Modificado: 20-03-20

Aceptado: 27-03-20

\section{Bibliografía}

1. Rivera-Hernández F. Biopsia renal. Sección de Nefrología. Hospital General de Ciudad Real, en línea. NefroPlus 2009;2(1):1-8. 
2. Serón-Micas D. Biopsia renal. [Consultado el 03 de Diciembre del 2019]. Disponible en https://hospital. vallhebron.com/es/pruebas-diagnosticas/biopsia-renal.

3. Alcaraz-Guirado AJ; Alcaraz-Guirado A; Sánchez-Joya MM. Biopsias. En: Tratado enfermería cuidados críticos, pediátricos y neonatales, en línea [Internet]. Actualización Octubre 2016. [Consultado 09 Abr de 2019]. Disponible en: https://ajibarra. org/D/post/capitulobiopsias/.

4. Mayor M, Troche A, Martínez Pico M, Matsumura $K$, Nunes Velloso MC. Características clínicas y anatomopatológicas de pacientes sometidos a Biopsia Renal en una Sala de Nefrología Pediátrica. Pediatr.(Asunción) 2009;36(1):29-34.

5. Álvarez-Suarez JL, Castillo-Arévalo F, Fernández-Fidalgo $D$, Muñoz-Meléndez M. Manual de Valoración de Patrones Funcionales. Servicio de Salud del Principado de Asturias. Atencion Primaria Area V Gijon [(Internet]. [Consultado 30 Nov 2019]. Disponible en: https://seapaonline.org/UserFiles/ File/Ayuda\%20en\%20consulta/MANUAL\%20VALORACION\%20NOV\%202010.pdf.
6. Heather T, Kamitsuru S, Editores: Diagnosticos enfermeros. Definiciones y clasificación 2015-2017. Barcelona: Elsevier España SL; 2015.

7. Moorhead S, Swanson E; Johnson M, Maas M. Clasificación de Resultados de Enfermería (NOC). Medición de Resultados en Salud. $5^{a}$ edición. Barcelona: Elselvier España SL; 2014.

8. Bulechek GM, Butcher HK, Dochterman JM, Wagner CHM. Clasificación de Intervenciones de Enfermería (NIC). $6^{a}$ edición. Barcelona: Elselvier Espaก̃a SL; 2014.

9. Toledo K, Perez-Sáez MJ, Espinosa M, Gomez J, Lopez M, Redondo D. Complicaciones asociadas a la biopsia renal percutánea. Experiencia en España 50 años después. Nefrología. 2010;30(5):487-98.

10. Rivera-Hernandez F. Biopsia renal. Nefrología al día. Hospital General Universitario de Ciudad Real. [Internet] Actualización Diciembre 2019. [Consultado 15 dic 2019]. Disponible en: https://www.nefrologiaaldia.org/es-articulo-biopsia-renal-234.

Este artículo se distribuye bajo una Licencia Creative Commons Atribución-NoComercial 4.0 Internacional. https://creativecommons.org/licenses/by-nc/4.0/ 